\title{
Robust Stabilizing Controllers to Automatic Generation Control for Load Frequency Control Application
}

\author{
M. R. I. Sheikh ${ }^{1}$, R. Takahashi, and J. Tamura \\ Kitami Institute of Technology, 165 Koen-cho, Hokkaido, Kitami, Japan \\ Received 16 August 2009, accepted in final revised form 2010
}

\begin{abstract}
Since superconducting magnetic energy storage (SMES) unit with a self-commutated converter is capable of controlling both the active and reactive powers simultaneously and quickly, increasing attention has been focused recently on power system stabilization by SMES control. This study presents the effects of novel control strategies of self-tuned fuzzy proportional integral (FPI) controller and fuzzy frequency (FF) controller associated with the automatic generation control (AGC) including SMES unit. The effects of the self-tuning configuration with FPI controller in AGC is also compared with that of FF controlled AGC on SMES control. The simulation results show that both self tuning control schemes of AGC are very effective in damping out of the oscillations caused by load disturbances and it is also seen that the FF controlled AGC with SMES perform better primary frequency control compared to FPI controlled AGC with SMES.
\end{abstract}

Keywords: Load frequency control; Single area power system; FPI controller; FF controller; SMES unit.

C) 2010 JSR Publications. ISSN: 2070-0237 (Print); 2070-0245 (Online). All rights reserved.

DOI: 10.3329 /jsr.v2i2.3063

J. Sci. Res. 2 (2), 285-293 (2010)

\section{Introduction}

Automatic generation control is a very important subject in power system operation for supplying sufficient and reliable electric power. In an interconnected power system, due to the variation of load demand, the area frequency and tie-line power interchange also vary randomly. The LFC by only a governor control imposes a limit on the degree to which the deviations in frequency and tie-line power exchange can be decreased. However, as the LFC is fundamentally for the problem of an instantaneous mismatch between the generation and demand of active power, the incorporation of a fast-acting energy storage device in the power system can improve the performance under such

${ }^{1}$ Corresponding author: ris_ruet@yahoo.com 
conditions. To achieve a better performance, many control strategies are proposed in literature [1-3]. But fixed gain controllers based on classical control theories are presently used. These are insufficient because of changes in operating points during a daily cycle and are not suitable for all operating conditions. Therefore, variable structure controllers [4-5] are proposed for AGC. For designing these control techniques, the perfect model is required which has to track the state variables and satisfy system constraints. So it is difficult to apply these adaptive control techniques to AGC in practical implementations. In multi-area power system, if the load variation occurs at any one of the areas in the system, the frequency related with this area is affected first and then that of the other areas are also affected from this perturbation through tie lines. But in single area power system, the frequency is affected directly from any type of perturbation. When a small load disturbance occurs, power system frequency oscillations continue for a long duration, even in the case with optimized gain of integral controllers [6]. So, to damp out the oscillations in the shortest possible time, automatic generation control including SMES unit is used.

In the proposed self-tuning technique, the controllers are so designed that it compromise between fast transient recovery and low overshoot in dynamic response of the system. For this, the frequency deviation $(\Delta f)$ is used as the control input to SMES controller. It is seen that both FPI and FF control schemes of AGC are very effective in damping out of the oscillations caused by load disturbances and it is also seen that the FF controlled AGC with SMES perform more effective primary frequency control compared to FPI controlled AGC with SMES. Considering these viewpoints, this study presents the novel control strategies for AGC including SMES.

\section{Power System Model for AGC Including SMES}

The single area power system model with the proposed configuration of SMES unit is shown in Fig. 1. When there is a sudden rise in power demand in a control area, the stored energy is almost immediately released by the SMES through it's power conversion system. As the governor control mechanism starts working to set the power system to the new equilibrium condition, the SMES coil stores back to its nominal energy level. Similar action also happens when there is a sudden decrease in load demand.

Basically, the governor-turbine system is slow reacting compared with the excitation system, which is fast reacting. As a result, fluctuations in terminal voltage can be corrected by the excitation system very quickly [3]. Fluctuations in generated power or frequency are corrected slowly. Since load frequency control is primarily concerned with the real power/frequency behavior, the excitation system model will not be required in the approximated analysis [3]. This important simplification paves the way for the required digital simulation analyses of the model system shown in Fig. 1. Detail descriptions about DB and GRC are available in ref. [7]. All parameters are same as those used in [8]. 


\section{Optimization of the Integral Gain, $\mathrm{K}_{\mathrm{I}}$ for Fixed gain PI Controller}

The tuning of the value of $K_{I}$ at $K p=0$ was achieved using a systematic exhaustive search according to the IAET criterion shown in Eq. (1).

$$
J_{f r e}=\int_{0}^{T}|\Delta f(t)| t d t
$$

Considering this performance index $\left(J_{\text {fre }}\right)$ for the fixed load disturbance, the optimal value of fixed gain $K_{I}$ is determined for the fixed gain controller. It is found that in the absence of generation rate constraints (GRC) the best-tuned integral gain value is $K_{I}=$ 0.29 and $K_{P}=0$ at $J_{f r e}=0.1871$, which is also called the critical value. In the presence of governor dead-band (DB) and GRC the gain values of the conventional PI controller are $K_{I}=0.27$ and $K_{P}=0$ at $J_{f r e}=0.2696$, which is shown in Fig. 2.

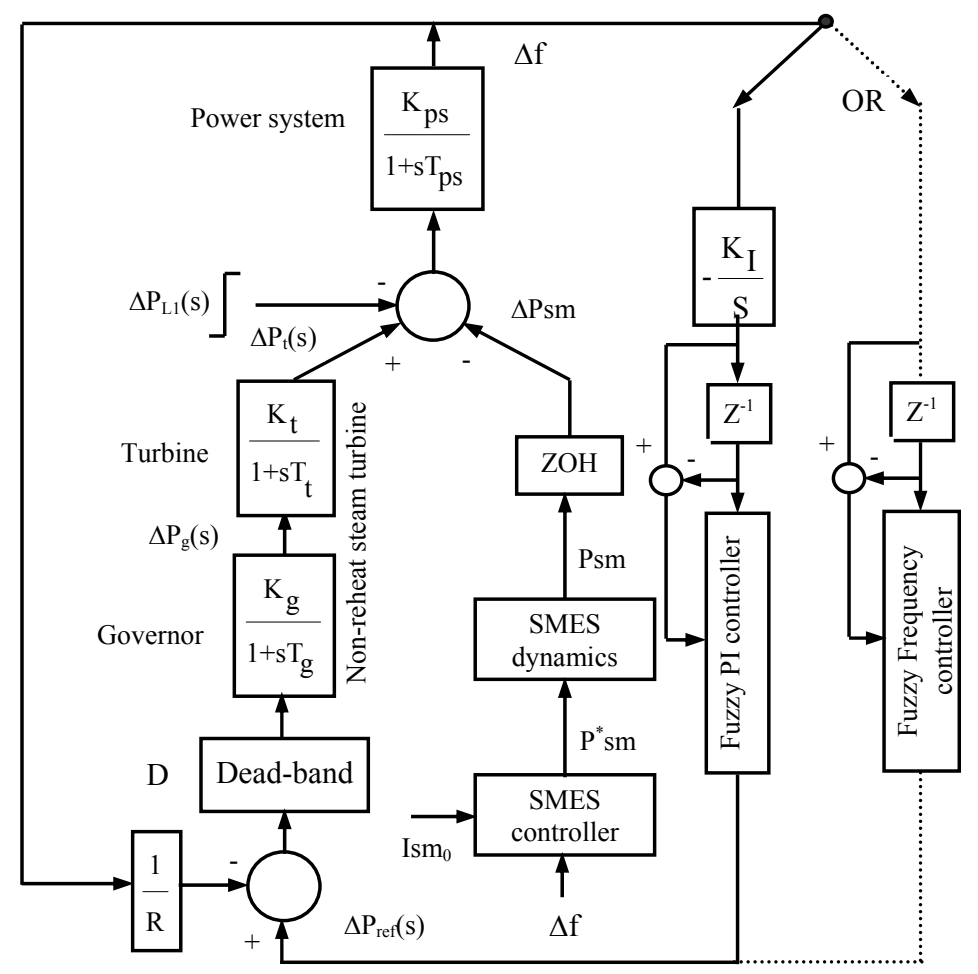

Fig. 1. Power system model with SMES. 


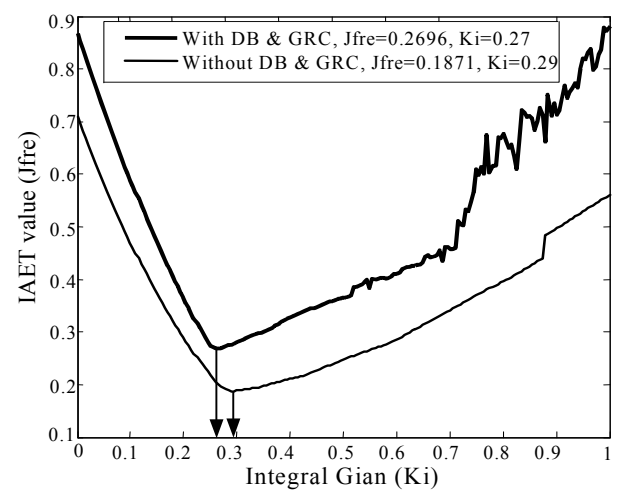

Fig. 2. The optimal $\mathrm{K}_{\mathrm{I}}$ setting with and without considering DB and GRC.

\section{Design of Fuzzy Frequency (FF) Controller}

The fuzzy frequency controller has the two inputs, which are defined as:

Input 1: $\quad$ error $=e_{t}=\Delta f=f_{\text {norm }}-f_{t}$,

Input 2: rate of change of error $=c \dot{e}_{t}=\dot{\Delta} f=\dot{f}_{\text {nominal }}-\dot{f}_{t}$,

The triangular membership functions for the proposed FF controller of the three variables $\left(e_{t}, c \dot{e}_{t}, \Delta P_{r e f}\right)$ are shown in Fig. 3, where error, $e_{t}$, and change of error, $c \dot{e}_{t}$, are used as the inputs of the fuzzy logic controller. Considering these two inputs, the output of FF controller, $\Delta P_{\text {ref }}$, is determined. The use of two input and single output variable makes the design of the controller very straightforward. A membership value for the various linguistic variables is calculated by the rule given by

$$
\mu\left(e_{t}, c \dot{e}_{t}\right)=\min \left[\mu\left(e_{t}\right), \mu\left(c \dot{e}_{t}\right)\right]
$$

The equation of the triangular membership function used to determine the grade of membership values in this work is as follows:

$$
A(x)=\frac{(b-2|x-a|)}{b},
$$

where $A(x)$ is the value of grade of membership, ' $\mathrm{b}$ ' is the width and ' $\mathrm{a}$ ' is the coordinate of the point at which the grade of membership is 1 and $x$ is the value of the input variables. The control rules for the proposed strategy are very straightforward and have been developed from the viewpoint of practical system operation and by trial and error methods. The fuzzy rule base for the FF control scheme is shown in Table 1. 
The membership functions, knowledge base and method of defuzzification determine the performance of the FF controller in a single area power system as shown in Eq.(6). Detail description of FPI controller is also available in [9].

$$
\Delta P_{r e f}=\frac{\sum_{j=1}^{n} \mu_{j} u_{j}}{\sum_{j=1}^{n} \mu_{j}},
$$
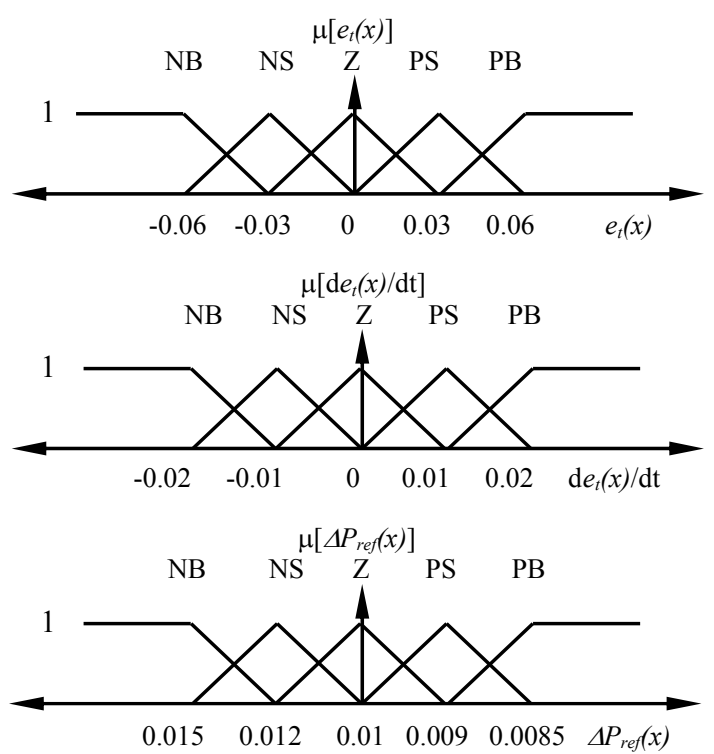

Fig. 3. Membership functions for the fuzzy variables.

Table 1. Fuzzy rule base for FF controller.

\begin{tabular}{llllll}
\hline & NB & NS & Z & PS & PB \\
\hline NB & PB & PB & PS & PS & Z \\
NS & PB & PS & PS & Z & NS \\
Z & PB & PS & Z & NS & NS \\
PS & PS & Z & NS & NS & NB \\
PB & Z & NS & NS & NB & NB \\
\hline
\end{tabular}

\section{Control Scheme of SMES}

\subsection{Brief overview of SMES}

The schematic diagram in Fig. 4 shows the configuration of a thyristor controlled SMES unit, which is incorporated in the power system for LFC application. The converter 
impresses positive or negative voltage on the superconducting coil. Charge and discharge are easily controlled by simply changing the delay angle $\alpha$, which controls the sequential firing of the thyristors. If $\alpha$ is less than $90^{\circ}$, the converter operates in the rectifier mode (charging) and if $\alpha$ is greater than $90^{\circ}$, the converter operates in the inverter mode (discharging). As a result, power can be absorbed from or released to the power system according to the system requirement. At steady state SMES should not consume any real or reactive power.

The assumptions given below are considered in modeling of the present SMES unit [10]:

(i) The superconducting coil has a large inductance so that the effect of ripple of the direct current is ignored.

(ii) The resistance of the superconducting coil is zero.

(iii) The voltage drop in the converter thyristor is ignored.

(iv) Harmonic power generated by the converter is neglected.

\subsection{Control system of SMES unit}

Fig. 5 outlines the proposed control scheme for SMES, which was designed to reduce the instantaneous mismatch between demand and generation. As the operating point changes due to load change, both FPI and FF controllers are proposed. Firstly, optimized value of $K_{I}$ is determined. Then output of FF or FPI controller, $\Delta P_{\text {ref, }}$ is determined using fuzzy logic controller to determine frequency deviation, $\Delta f$ and this $\Delta f$ is used as the input to the SMES controller. It is desirable to restore the inductor current to its rated value as quickly as possible after a system disturbance, so that the SMES unit can respond properly to any subsequent disturbance. So inductor current deviation is sensed and used as negative feedback signal in the SMES control loop to achieve quick restoration of current and SMES energy level. The parameters of the SMES controller are same as those used in [8].

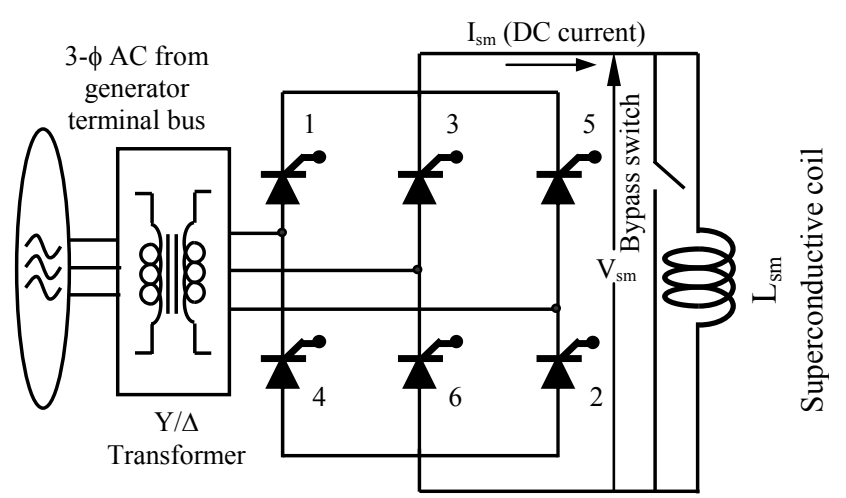

Fig. 4. SMES unit with 6-pulse bridge AC/DC thyristor controlled converter. 


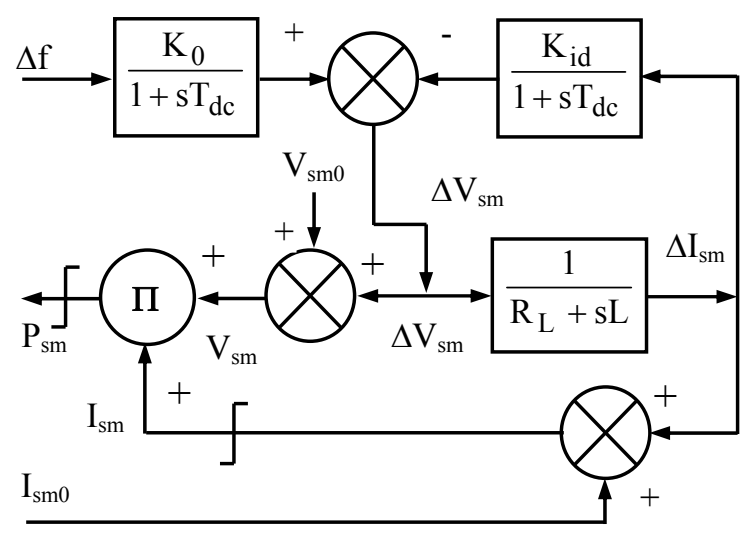

Fig. 5. SMES control system.

\section{Simulation Results and Discussions}

To demonstrate the beneficial damping effect of the proposed controller, computer simulations have been carried out for different load changes using the MATLAB environment. The system performances with FF and FPI controlled AGC including SMES are shown in Fig. 6 and Fig. 7. System performances without SMES are also shown in the same figure. Two cases studies are conducted.

Case I: When DB and GRC are not considered and investigate the effectiveness of the controllers with and without SMES.

Case II: When DB and GRC are considered and investigate the effectiveness of the controllers with and without SMES.

Fig. 6 and Fig. 7 show the system performances for both the cases with a step load increase of $\Delta P_{L}=0.025$ p.u.(50MW). For both the cases, it is seen from Fig. 6 that the self-tuning FF controller (FFC) and FPI controller of AGC including SMES are very effective in damping out of the oscillations caused by load disturbances compared to optimized fixed gain controller. Finally, it is seen from the results (Fig. 6) that when not considered DB and GRC, the proposed FFC scheme including SMES perform better primary frequency control than FPI controller including SMES. For Case II, Fig. 6 shows that system is completly unstable with only optimized fixed gain controller. It is also interesting to observe from Fig. 7 that $\mathrm{P}_{\mathrm{sm}}$ becomes zero and inductor current $\left(I_{\mathrm{sm}}\right)$ returns back to the rated value more quickly after providing appropriate compensation with FFC scheme. This enables the SMES unit to respond to a subsequent load disturbance in the power system more quickly. It is also observed that the deviation of inductor current, $\mathrm{I}_{\mathrm{sm}}$ and real power compensation, $\mathrm{P}_{\mathrm{sm}}$ are more less, in Case $\mathrm{I}$, when the proposed FFC scheme is used. The frequency deviations restore to its nominal value more quickly with the proposed FFC in AGC than that with the FPI controlled AGC including SMES, for Case I. Finally, it is concluded that FFC scheme can be used for both the cases for effective use of LFC application. 

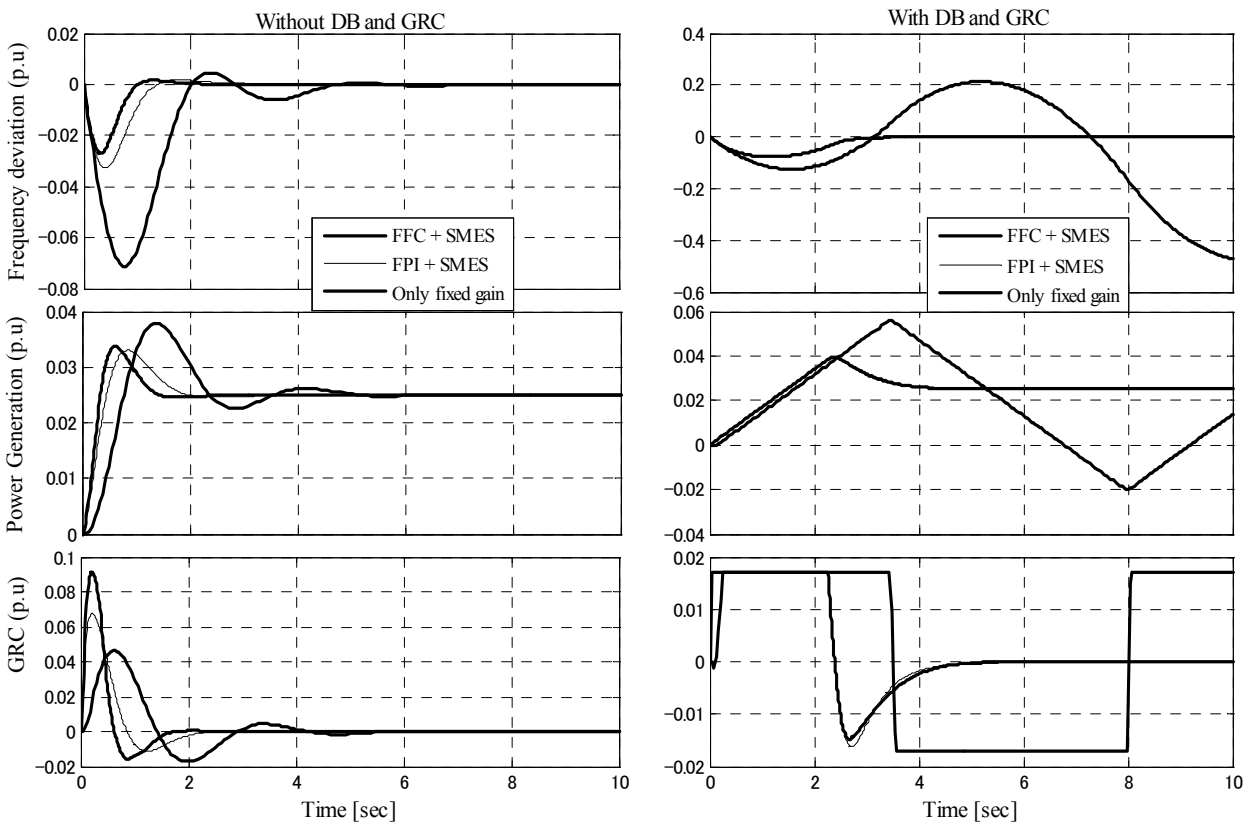

Fig. 6. System performances for a step load increase $\Delta P_{L}=0.025$ p.u.
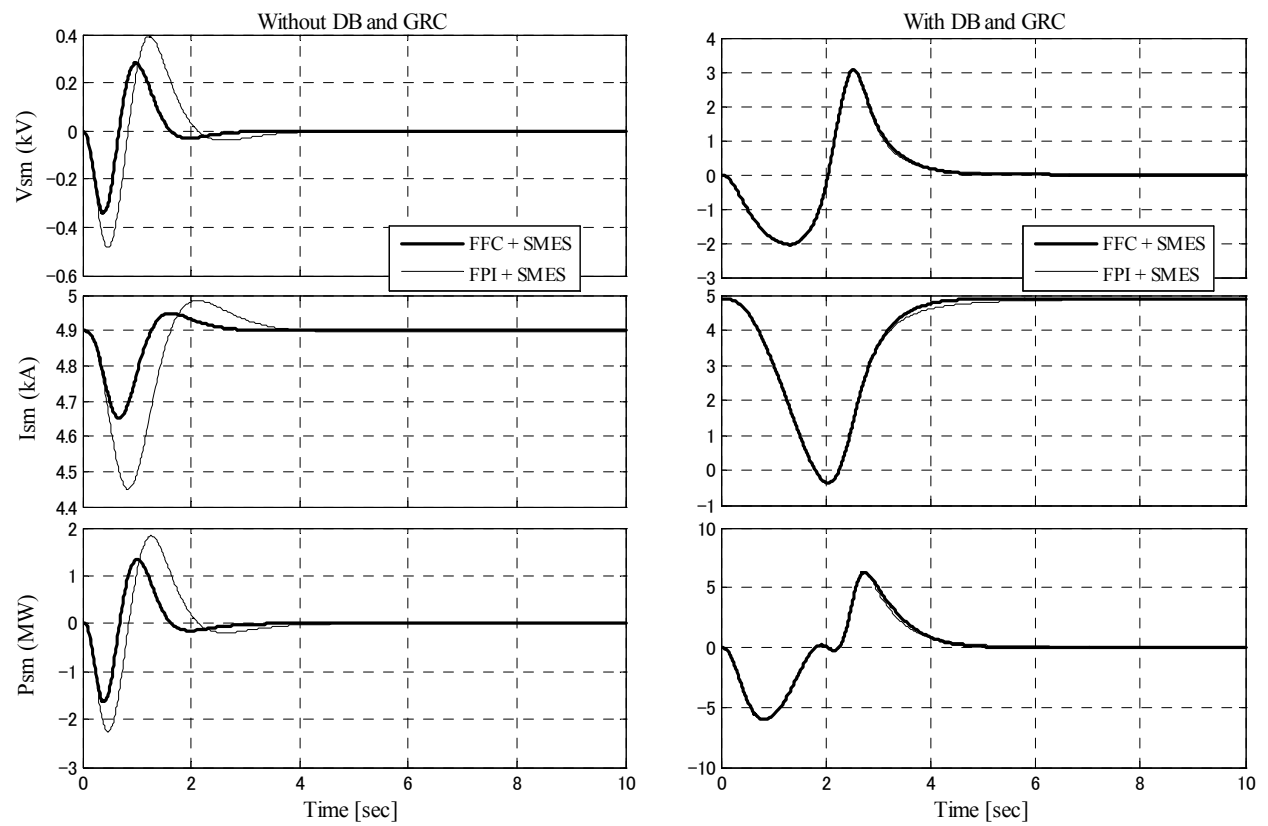

Fig. 7. System performances for a step load increase $\Delta P_{L}=0.025$ p.u. 


\section{Conclusions}

Two alternative and successful intelligent controllers are designed for AGC including SMES unit for LFC of single area power system. Both the FPI control and FF control approach yields automatic, self-adjusting outputs irrespective of widely varying, imprecise, uncertain off-nominal conditions. It is seen that with both FPI and FF controlled AGC, the simple SMES controller can compensate frequency deviation in the effective manner compared to optimized fixed gain controller. Finally, the simulation results show that the proposed FF controller is very powerful compared to FPI controller, with smaller inductor current deviation and lower SMES power compensation. Because of the adaptive feature, the more effective FF controller is proposed finally for AGC including SMES unit.

\section{References}

1. J. Nanda and B. L. Kavi, Automatic Generation Control of Interconnected Power System- Proc. IEE Gen., Trans. and Dist. 125, 385 (1988).

2. J. Nanda, A. Mangla, and S. Suri, IEEE Trans. on Energy Conv. 21, 187 (2006). doi:10.1109/TEC.2005.853757

3. M. U. Mufti, S. A. Lone, S. J. Iqbal, and I. Mushtaq, IEEJ Trans. 2, 387 (2007).

4. A. Y. Sivaramaksishana, M. V. Hariharan, and M. C. Srisailam, Int. J. of Control 40, 437 (1984).

5. S. C. Tripathy and K. P. Juengst, IEEE Trans. on Energy Conv. 12, 187 (1997). doi: $10.1109 / 60.629702$

6. M. R. I. Sheikh, S. M. Muyeen, R. Takahashi, T. Murata, and J. Tamura, ICEM, 08, Paper ID1026, (September 2008, Portugal).

7. M. R. I. Sheikh, S. M. Muyeen, R. Takahashi, T. Murata, and J. Tamura, $4^{\text {th }}$ Int. Conf. of Elec. \& Com. Eng. 1, 1 (2008).

8. M. R. I. Sheikh, and M. G. Rabbani, Application of a Self-Tuning Fuzzy Gain Scheduled Controller to AGC in Power System Including SMES Unit, Accepted for publication in SEUJSE (2010).

9. M. R. I. Sheikh, S. M. Muyeen, R. Takahashi, T. Murata, and J. Tamura, Application of SelfTuning FPIC to AGC for Load Frequency Control in Multi-Area Power System, 2009 IEEE Bucharest Power Tech Conf. (Bucharest, Romania, July 2009).

10. M. H. Ali, T. Murata, and J. Tamura, IEEE Trans. on Control Sys. Tech. 15, 144 (2007).

DOI: $10.1109 /$ TCST.2006.883342 